\title{
Vision-based Localization and Positioning of an AUV
}

\author{
André B. Figueiredo*†, Bruno M. Ferreira*, Aníbal C. Matos*† \\ * INESC TEC \\ $\dagger$ Faculty of Engineering, University of Porto \\ FEUP Campus \\ Rua Dr. Roberto Frias \\ 4200-465 Porto, Portugal
}

\begin{abstract}
This paper presents the last developments towards vision-based target tracking by an AUV. The main concepts behind the visual relative localization are provided and the results from a statistical analysis for the relative localization algorithm are presented. The purpose of this analysis is to ensure properness of data used to feed controllers that are responsible for governing the AUV motion. A new set of controllers enabling the AUV to track a visual target is given. Experimental data from obtained from tests in tank are presented, validating both the visual relative localization and control of the AUV.
\end{abstract}

\section{INTRODUCTION}

The work herein presents a method for relative localization of underwater autonomous robots based on artificial vision, contributing towards fully automatic missions of marine vehicles. This work follows on from our previous work [1] on which the vehicle was constrained to a constant relative angular position, which led us to a $2 \mathrm{D}$ positioning. In the present paper, we consider the relative navigation of an Autonomous Underwater Vehicle (AUV) with respect to coloured beacons in an unconstrained relative pose. In underwater robotics, relative positioning is an important field where several researchers have been applying considerable efforts. Our paper topic is located in two areas: computer vision and underwater robotics research. It draws on computational low cost techniques of image processing for visual perception and tracking methods and, on the other hand, on relative navigation techniques. The visual system for a totally autonomous close-range tracking and positioning process presented in this work is integrated as a module in the MARES AUV system. MARES is a torpedo shaped AUV developed by CROB Laboratory at INESC TEC. For the purpose of visual data collecting, the AUV MARES is equipped with a single camera installed on vehicle's bow.

\section{ARTIFICIAL VISION}

Cameras are in general considered cheaper solutions with richer information and providing superior update rates when compared to other commonly used sensors in robotics. Nevertheless, for the case of underwater robotics, the range of vision sensors is limited because of the poor visibility in underwater environments, which is a significant constraint that has to be carefully handled. Although the range constrains the use of vision sensors in underwater environments, cameras are effective sensors for short range situations as they provide meaningful information at relatively high rates. Our current approach considers three artificial markers precisely placed on a support frame, which can be either static or mobile. Our system comprises a monocular artificial vision sensor, placed on the vehicle, and three artificial coloured spheres (with different colors), mounted on a support frame (see figure 2 ). In order to estimate the vertical relative distance and the horizontal relative position with respect to the support platform, the three coloured spheres are used as reference markers. In order to track the target in underwater conditions it is necessary to robustly detect the desired coloured spheres and on the other hand, estimate the relative position, both horizontal and vertical, and estimate the relative orientation between the vehicle and the support frame. The proposed approach for coloured spheres detection is based on a image color segmentation process.

Each captured frame is converted from RGB to HSV color space and then we threshold it for the range of the reference color. In order to eliminate some possible outliers, only the largest contour area is consider on the resultant segmented binary image. The final step is then to determine the center of mass for that contour. This process is repeated until we find, in each frame, the center of mass of the three different reference colors of the spheres. With three points identified in a frame we can proceed to relative position and attitude estimation.

To the propose of estimate the position and attitude of the AUV Mares relatively to the target, we have adapted the algorithm described on [2].

The coordinates of the target's reference marks are related to the AUV's camera fixed coordinates by the following equation:

$$
\left[\begin{array}{lll}
x_{c} & y_{c} & y_{c}
\end{array}\right]=\left[A_{c t}\right]\left[\begin{array}{lll}
x_{t} & y_{t} & y_{t}
\end{array}\right]^{T}+[d]
$$

Where $\left[A_{c t}\right]$ is a $3 \times 3$ orthogonal matrix representing the relative orientation of the AUV Mares with respect to target body frame and $[d]$ is a $3 \times 1$ column vector denoting the relative position of feature points with respect to the camera focal point. The attitude matrix $\left[A_{c t}\right]$ is represented in terms of the quaternion vector $\left[\begin{array}{llll}q_{1} & q_{2} & q_{3} & q_{4}\end{array}\right]^{T}$ as 


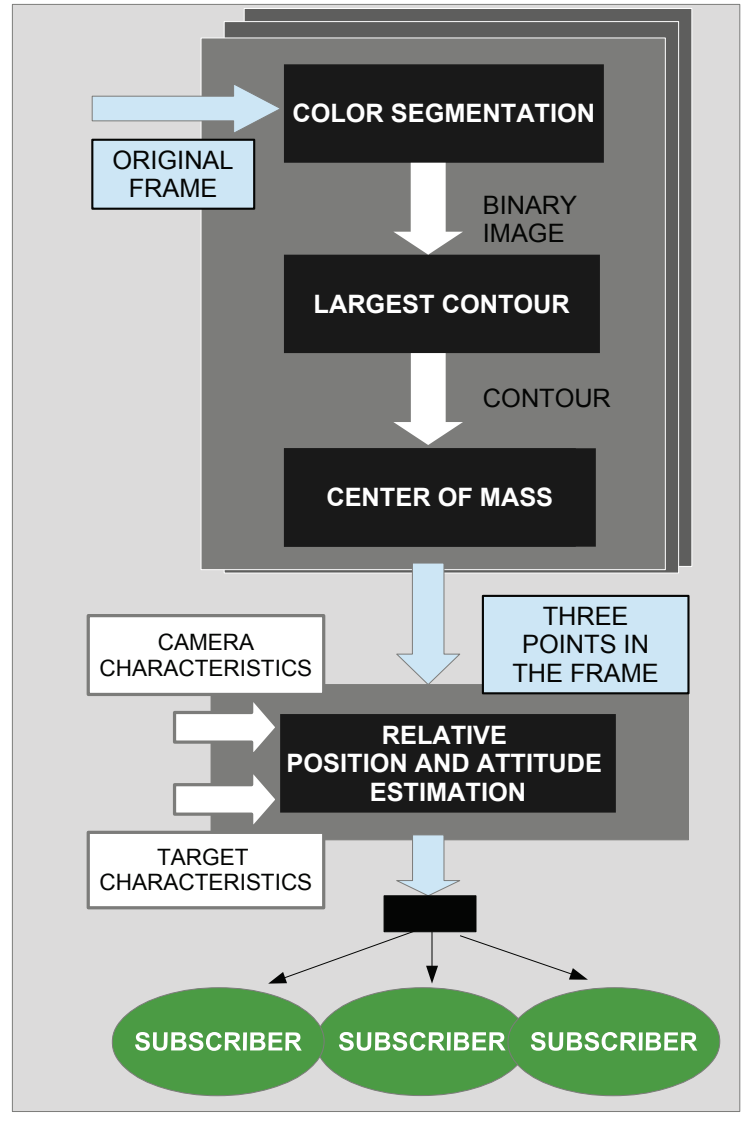

Fig. 1. Proposed approach for the coloured spheres detection

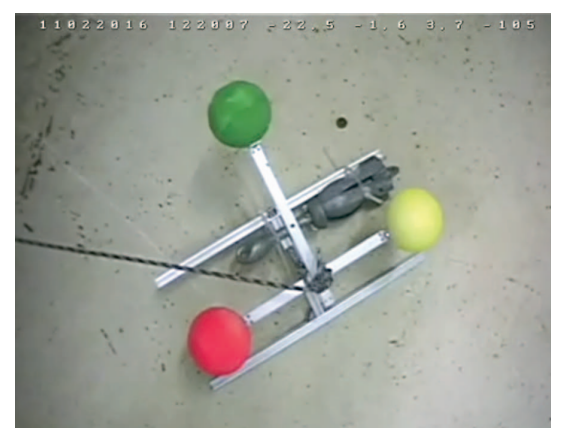

Fig. 2. target: three coloured spheres on a support frame

$$
\begin{aligned}
{\left[A_{c t}\right]=\left[\begin{array}{cc}
\left(q_{1}^{2}-q_{2}^{2}-q_{3}^{2}+q_{4}^{2}\right) & 2\left(q_{1} q_{2}+q_{3} q_{4}\right) \\
2\left(q_{1} q_{2}-q_{3} q_{4}\right) & \left(-q_{1}^{2}+q_{2}^{2}-q_{3}^{2}+q_{4}^{2}\right) \\
2\left(q_{1} q_{3}+q_{2} q_{4}\right) & 2\left(q_{2} q_{3}-q_{1} q_{4}\right) \\
2\left(q_{2} q_{4}-q_{1} q_{3}\right) \\
2\left(q_{2} q_{3}+q_{1} q_{4}\right) \\
& \left(-q_{1}^{2}-q_{2}^{2}+q_{3}^{2}+q_{4}^{2}\right)
\end{array}\right] }
\end{aligned}
$$

The relative position vector $[d]$ is represented as

$$
d=\left[\begin{array}{lll}
x_{r} & y_{r} & z_{r}
\end{array}\right]^{T}
$$

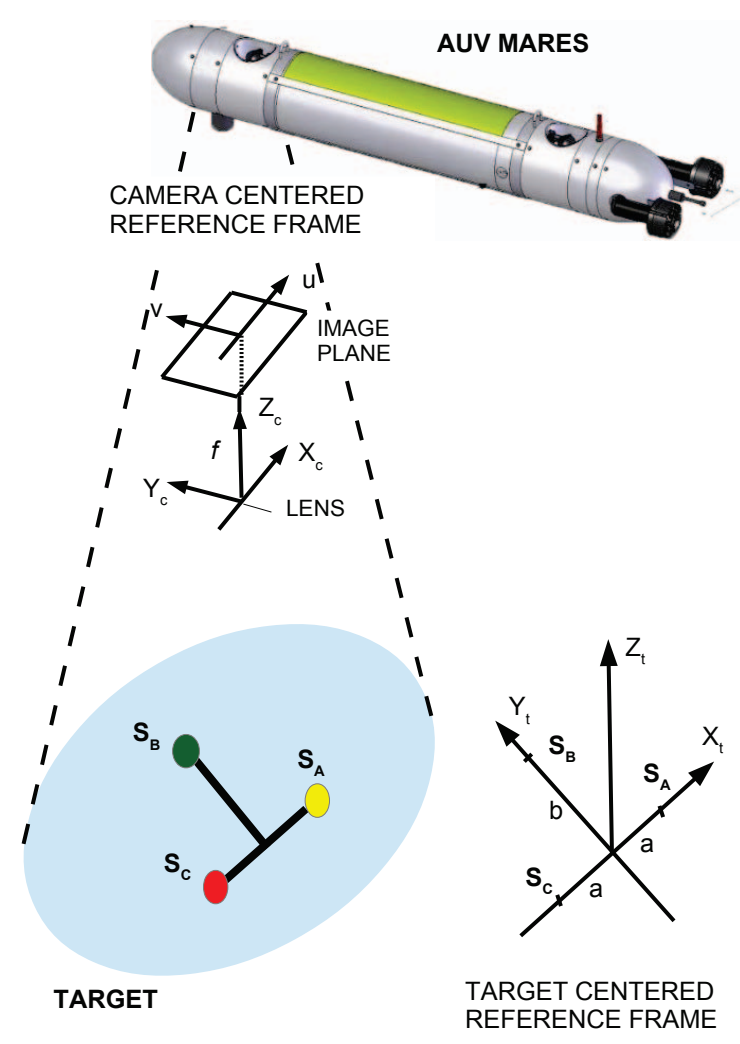

Fig. 3. Definitions of the camera and target reference frames

where $x_{r}, y_{r}, z_{r}$ are the relative distances of the centre of the feature points from the camera along $x_{c}, y_{c}, z_{c}$ directions. The position of feature points on the target are defined as

$$
\begin{gathered}
S_{A}:\left[\begin{array}{l}
x_{A} \\
y_{A} \\
z_{A}
\end{array}\right]=\left[\begin{array}{l}
a \\
0 \\
0
\end{array}\right] ; \\
S_{B}:\left[\begin{array}{l}
x_{B} \\
y_{B} \\
z_{B}
\end{array}\right]=\left[\begin{array}{c}
0 \\
-b \\
0
\end{array}\right] ; \\
S_{C}:\left[\begin{array}{l}
x_{C} \\
y_{C} \\
z_{C}
\end{array}\right]=\left[\begin{array}{c}
-a \\
0 \\
0
\end{array}\right] ;
\end{gathered}
$$

1) Characterization of the developed vision-based module: Before proceeding to the interaction with the control strategy, the performance of the visual module was characterized. This characterization allowed us to have a notion about the reliability and consistency of the data produced by the module.

The camera was placed at a fixed position. Then using tube rails, the target was moved along the $\mathrm{Z}$ and $\mathrm{X}$ axis and positioning at well known positions. At each position, the target was rotated in Yaw and Roll angles. 
To observe the effect of moving the target in $\mathrm{Y}$ axis, the camera was turned from landscape orientation to portrait orientation. The previous process of moving the target was repeated.

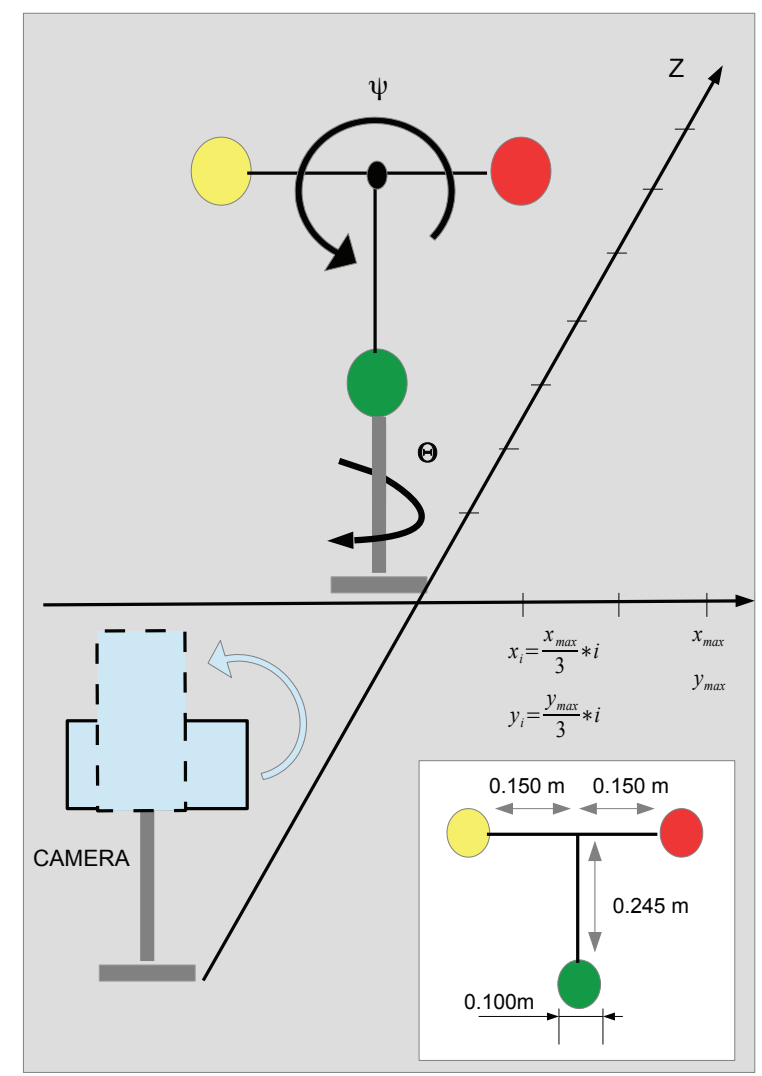

Fig. 4. The procedure used to characterize the visual module

The figures 4 and 5 illustrate the followed characterization procedure.

The figures 6 and 7 summarize the obtained characterization results when the target was moved along the $\mathrm{Z}$ axis.

The figure 6 gives us the notion about the system precision based on standard deviation analysis. This figure shows the standard deviation evolution for the three axis when the target was moved away from the camera along the $\mathrm{Z}$ axis. In average the standard deviation in $\mathrm{X}$ and $\mathrm{Y}$ axis was about 2 millimetres, and in $\mathrm{Z}$ was about 15 millimetres.

The Fig. 7 lets us appreciate the mean values of the estimated distance from the camera to the target in the three axis. The variations in $\mathrm{X}$ and $\mathrm{Y}$ axes is due to the nonexistence of a mechanical solution which guarantees the correct alignment between the centre of the camera to the tube rail. This misalignment has influence especially on the $\mathrm{X}$ axis, which means a yaw rotation of the camera related to the rail. The increasing offset along the $\mathrm{Z}$ axis is related to the non calibration of the value of the focal length of the camera's lens.

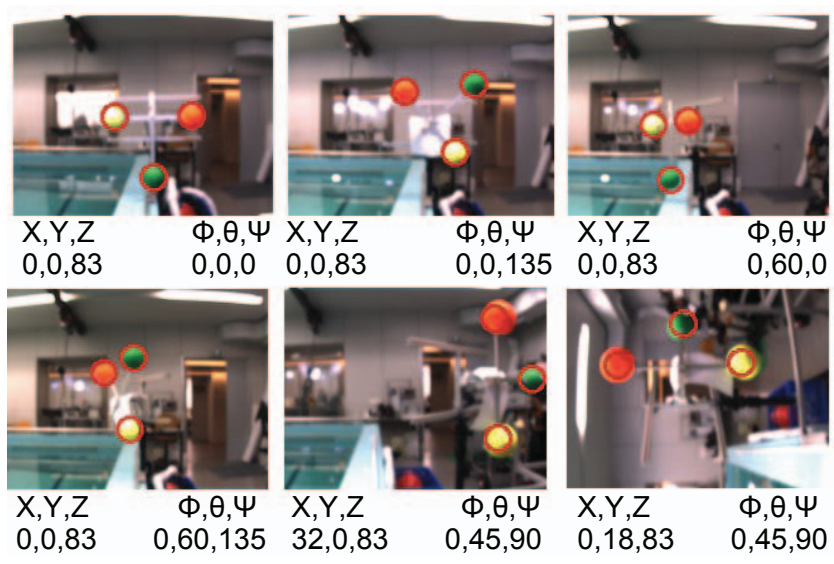

Fig. 5. The procedure used to characterize the visual module
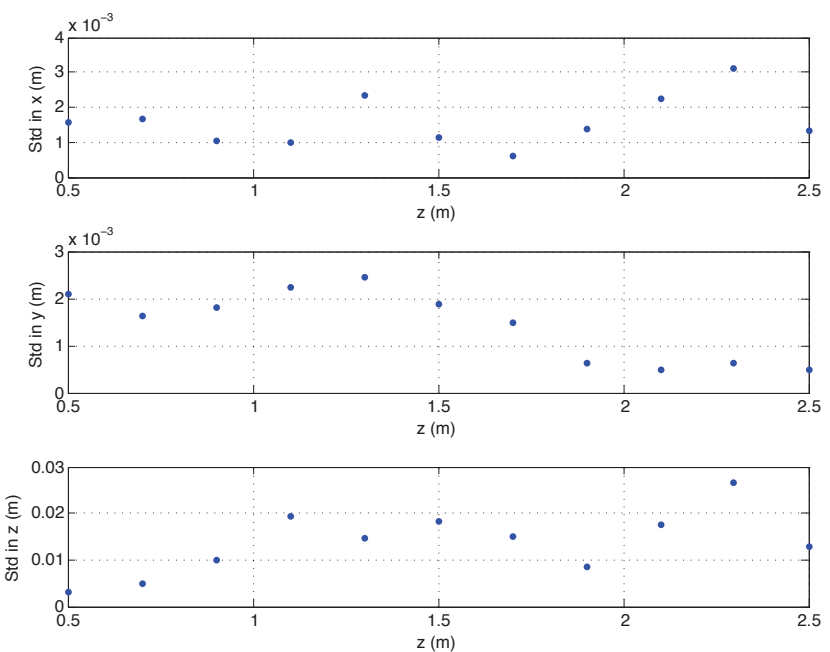

Fig. 6. Standard deviation analysis: moving the target away from the camera along the $\mathrm{Z}$ axis

To avoid these systematic observed errors is required a precise mechanical rail solution and a lens's camera calibration.

The table I summarizes the obtained characterization results when the target was moved along the $\mathrm{X}$ axis.

The offset observed, in the table I, between $x$ and mean $x$ is related to the misalignment of the camera related to the rail. Despite of this fact, the standard deviation is bellow 8 millimetres in all axis.

The table II summarizes the obtained characterization results when the target was rotated in $\theta$ at a fixed point. (see figure 4)

TABLE I

FIXED Z=1.66M; MOVING IN X, 160 SAMPLES PER POSITIONS

$\begin{array}{lllll}\mathrm{x} & \mathrm{y} & \mathrm{z} & \text { mean } \mathrm{x} & \text { std } \mathrm{x} \\ 0.31 & 0 & 1.66 & 0.2606 & 0.0013 \\ 0.63 & 0 & 1.66 & 0.5696 & 0.0059 \\ 0.94 & 0 & 1.66 & 0.8844 & 0.0073\end{array}$



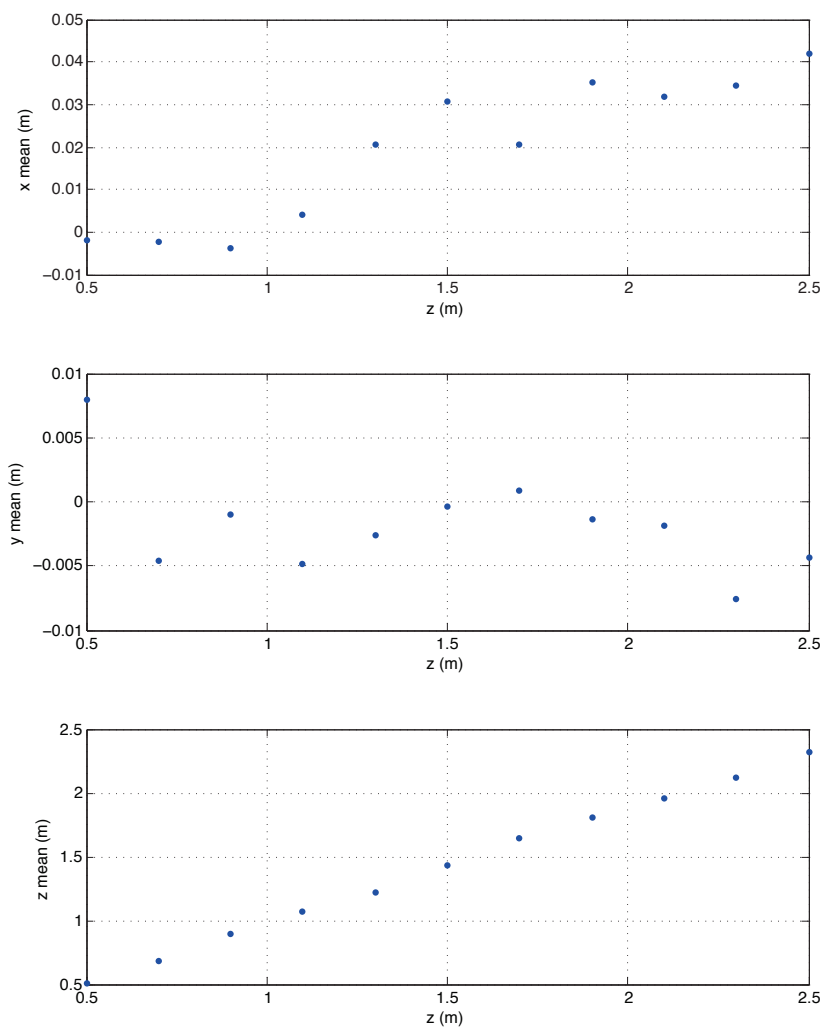

Fig. 7. Mean values analysis(160 samples per fixed position): moving the target away from the camera along the $\mathrm{Z}$ axis

TABLE II

FIXED $X=1.5 \mathrm{M}$; FIXED $\mathrm{Y}=0 \mathrm{M}$;FIXED $\mathrm{Z}=2.5 \mathrm{M}$; MOVING $\theta, 160$ SAMPLES PER $\theta$

$\begin{array}{llll}\theta & \text { mean x } & \text { mean y } & \text { mean } \mathrm{z} \\ 0 & 1.4665 & 0.0684 & 2.5992 \\ 45 & 1.4557 & 0.0098 & 2.6101\end{array}$

From the table II we can conclude the distance still observable when the target is rotated in 45 degrees.

In the whole characterization, the accuracy of the obtained values are in a reasonable range to proceed to the interaction with the control strategy.

\section{Control Strategy}

A control solution for position tracking is now proposed. Complementary to the vision-based localization, the vehicle must be able to position itself with regard to the target. The control strategy is built in a modular fashion where two control loops are responsible to drive the vehicle according to a predefined reference position. In the present work, this reference is a constant position fixed to the three artificial markers. At a lower level, a controller uses a parametrizable dynamics model to stabilize linear and angular velocities around a variable velocity reference. At an upper level a pose controller generates velocity references to guide the vehicle's pose. This strategy is better known in literature as inner-outer loop controller.
Beyond its versatility that enables modifying pose and velocity controllers independently, it also enables switching from a pose controller to another, depending on the maneuver to be executed. For example, line-tracking and station-keeping controllers at the pose (kinematics) level can use the same velocity controller at the velocity (dynamics) level, rather than designing all new, specific, control laws for these purposes.

The method is applied to MARES (fig. 8), a small-sized, modular AUV whose length is comprised between 1.5 to $1.8 \mathrm{~m}$ in its standard configuration. The vehicle weights approximately $38 \mathrm{~kg}$ and is equipped with four thrusters providing four degrees of freedom. The hovering capability, provided by its two vertical thrusters, is an important feature for stationkeeping with regard to the a static object.

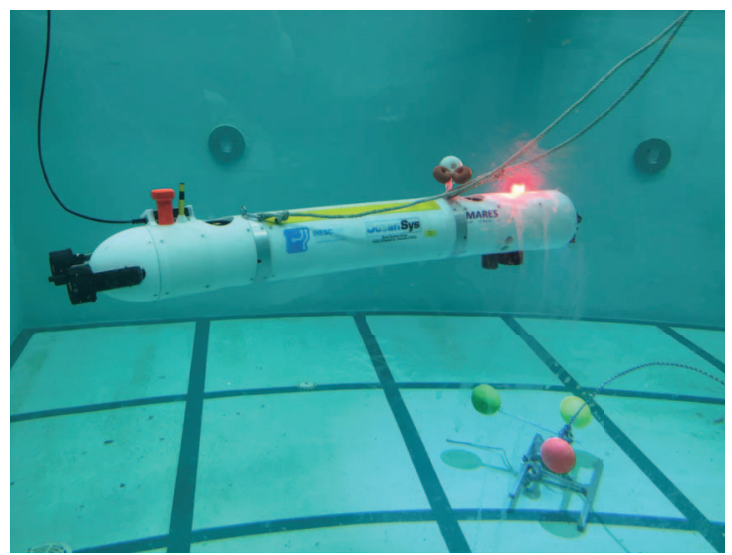

Fig. 8. The MARES AUV

\section{A. Vehicle's motion}

Consider a vehicle with $n_{c}$ controlled degrees of freedom (DOFs), whose pose is defined by a six-dimensional vector $\eta=\left[\begin{array}{ll}\eta_{l}^{T} & \eta_{a}^{T}\end{array}\right]$, expressed in an inertial, earth-fixed referential $\{\mathcal{I}\}$, where $\eta_{l}=\left[\begin{array}{lll}x & y & z\end{array}\right]^{T}$ is the vector of linear threedimensional position and $\eta_{a}=\left[\begin{array}{ll}\phi \theta & \psi\end{array}\right]^{T}$ is the vector of roll, pitch and yaw angles.

In order to simplify the following sections, a vehicle-fixed referential $\{\mathcal{B}\}$ was also considered, with regard to which the linear and angular velocities of the vehicle are expressed, as follows: $\nu=\left[\begin{array}{ll}\nu_{l} & \nu_{a}\end{array}\right]$, where $\nu_{l}=\left[\begin{array}{lll}u & v & w\end{array}\right]^{T}$ is the vector of linear surge, sway and heave velocities and $\nu_{a}=\left[\begin{array}{lll}p & q & r\end{array}\right]$ is the vector of angular rolling, pitching and yawing velocities, respectively.

The pose vector time derivative and the body-expressed velocity are related though the following expression:

$$
\dot{\eta}=J\left(\eta_{a}\right) \nu,
$$

where $J\left(\eta_{a}\right)$ is a mapping function between the frames $\{\mathcal{B}\}$ and $\{\mathcal{I}\}$, which can be decomposed as follows:

$$
J\left(\eta_{a}\right)=\left[\begin{array}{cc}
J_{l}\left(\eta_{a}\right) & \mathbf{0}_{3 \times 3} \\
\mathbf{0}_{3 \times 3} & J_{a}\left(\eta_{a}\right)
\end{array}\right] .
$$

For further details on these matrices, the reader is referred to [3]. 
The dynamics of a vehicle can be generically described by the nonlinear differential equation

$$
M \dot{\nu}=A(\nu) \nu-g(\eta)+T \tau,
$$

where $M \in \mathbb{R}^{6 \times 6}$ is the added mass and inertia matrix, the matrix $A \in \mathbb{R}^{6 \times 6}$ results from the hydrodynamic forces applied on the body of the vehicle when it is moving at a velocity $\nu$. The term $A(\nu) \nu$ constitutes the effect of added mass, Coriolis, centriptal and viscous damping forces and moments. The vector $g \in \mathbb{R}^{6}$ includes the effects of the restoring forces and moments, while $T \in \mathbb{R}^{6 \times n_{t}}$, with $n_{t} \geq n_{c}$, is a constant matrix that maps the forces and moments created by the $n_{t}$ actuators, whose actuation forces are given in the vector $\tau \in \mathbb{R}^{n_{t}}$, in the body-fixed reference frame.

\section{B. Vehicle's control}

1) Velocity control: In the decoupled control architecture presented here, different loops are considered where different quantities are stabilized at desired, possibly time-varying, set points. The goal now is to stabilizing the velocity vector at a desired velocity $\nu^{*} \in \mathbb{R}^{n}$, where $n \leq 6$. This vector, with a possibly lower dimension than the dynamics model (9), comes from the interest of controlling only part of the DOFs. In nonholonomic robots, the dimension of this vector is often equal to the number of DOFs, that is, the number of velocities that can be controlled directly. Sometimes, the dimension can be smaller than the degrees of freedom (DOFs), by neglecting some of them and using the fact that they are well-behaved or assuming that they are controlled independently (see, for example, [4]). The Lyapunov direct method will be used to derive the velocity control law.

Based on (9), consider the reduced order velocity vector $\bar{\nu}(t): \mathbb{R} \rightarrow \mathbb{R}^{n}$, whose dynamics is given by

$$
\dot{\bar{\nu}}=P M^{-1}(A(\nu) \nu-g(\eta)+T \tau)
$$

where the reduced order matrices and vectors are given by

$$
\bar{\nu}(t)=P \nu,
$$

with $P \in R^{n \times 6}$ being the projection matrix. This matrix maps the overall system in a smaller dimension subspace considering only the relevant DOFs, by selecting the corresponding rows and columns. A necessary condition is that the product $P M^{-1} T$ has full row rank.

In order to find a suitable control law, define the desired velocity vector as $\nu^{*} \in \mathbb{R}^{n}$ and the velocity error vector as:

$$
\tilde{\nu}=\bar{\nu}(t)-\nu^{*}
$$

which one desire to reduce to zero.

Hence, the control law is chosen as

$$
\tau=\left(P M^{-1} T\right)^{\dagger}\left(P M^{-1}(A(\nu) \nu-g(\eta))+\dot{\nu}^{*}-K_{\nu} \tilde{\nu}\right) .
$$

This choice guarantees exponential stability of the velocity.
2) Pose guidance: Assume that the AUV has three DOFs, namely surge, heave and yaw. Actually, MARES has an additional DOF, pitch, but using the current approach it may be decoupled from the remaining by applying an additional guidance law. For simplicity, it will not be presented here and it will be assumed that pitch is stable and that the vehicle is kept horizontal. Denote the desired position to be tracked $\tilde{\eta}_{B}$, expressed in the body coordinate system. In practice $\tilde{\eta}_{B}$ is directly obtained from relative localization with regard to the target, obtained from the perception and localization algorithms presented earlier. This same vector, expressed in the earth-fixed referential is expressed as $\tilde{\eta}=J_{l}\left(\eta_{a}\right) \tilde{\eta}_{B}$. It is assumed that the position of the desired position (target), expressed in the earth-fixed coordinate system, does not change over time.

The desired velocity vector takes the following form:

$$
\nu^{*}=\left[\begin{array}{lll}
u^{*} & w^{*} & r^{*}
\end{array}\right]^{T} \text {. }
$$

The guidance law for linear DOFs are chosen as follow:

$$
\begin{aligned}
& u^{*}=-P_{c}^{h} \frac{\tilde{\eta}_{B}}{\left\|H \tilde{\eta}_{B}\right\|}\left(-\xi_{l}^{h}-K_{\rho}^{h}\left(\left\|H \tilde{\eta}_{B}\right\|-\delta^{h}\right)\right) \\
& w^{*}=-P_{c}^{v} \frac{\tilde{\eta}_{B}}{\left\|V \tilde{\eta}_{B}\right\|}\left(-K_{\rho}^{v}\left(\left\|V \tilde{\eta}_{B}\right\|-\delta^{v}\right)\right)
\end{aligned}
$$

where $K_{\rho}^{(\cdot)}$ are positive gains, $\delta^{(\cdot)}$ are positive constants and

$$
\xi_{l}^{h}=\frac{1}{\left\|H \tilde{\eta}_{B}\right\|}\left(-\tilde{\eta}_{B}^{T} P_{u}^{h^{T}} P_{u}^{h} \nu_{l}\right)
$$

For clarity, the remaining matrices are given in section VI.

The desired yaw rate is given by

$$
\begin{aligned}
r^{*}= & B^{T}\left(B B^{T}\right)^{-1}\left(-\xi_{a}-K_{a} P_{u}^{h} \tilde{\eta}_{B}-\right. \\
& \left.\left(\left\|H \tilde{\eta}_{B}\right\|-\delta\right) P_{u}^{h} \frac{\tilde{\eta}_{B}}{\left\|H \tilde{\eta}_{B}\right\|^{2}}\left(\xi_{l}+K_{\rho}\left(\left\|H \tilde{\eta}_{B}\right\|-\delta^{h}\right)\right)\right)
\end{aligned}
$$

where $B=P_{u}^{h} S\left(\tilde{\eta}_{B}\right) N_{c}^{h^{T}}$ and $\xi_{a}=P_{u}^{h} S\left(\tilde{\eta}_{B}\right) N_{u}^{h^{T}} N_{u}^{h} \nu_{a}-$ $P_{u}^{h} J_{l}\left(\eta_{a}\right)^{T} \dot{\tilde{\eta}}$. The matrix $S$ is a skew symmetric matrix that takes the form

$$
S\left(\left[\begin{array}{lll}
\alpha_{1} & \alpha_{2} & \alpha_{3}
\end{array}\right]^{T}\right)=\left[\begin{array}{ccc}
0 & -\alpha_{3} & \alpha_{2} \\
\alpha_{3} & 0 & -\alpha_{1} \\
-\alpha_{2} & \alpha_{1} & 0
\end{array}\right] .
$$

The remaining matrices are also provided in section VI.

Note that these guidance laws suffer from singularities in certain conditions, namely when $\left\|H \tilde{\eta}_{B}\right\|=0,\left\|V \tilde{\eta}_{B}\right\|=0$ or $B=0$. Therefore, precautions must be taken to avoid such possibilities. The offset variables $\delta^{h}$ and $\delta^{v}$ must be designed so that the vehicle is sufficiently far from these conditions. The initial condition must also be considered.

It should be stressed that the AUV will be able to control relative position with regard to the target. However, the relative orientation cannot be decoupled from the position control and it comes as a function of the position error.

The complete derivation of these guidance laws and their proofs are beyond the scope of this paper and will not be presented here. 

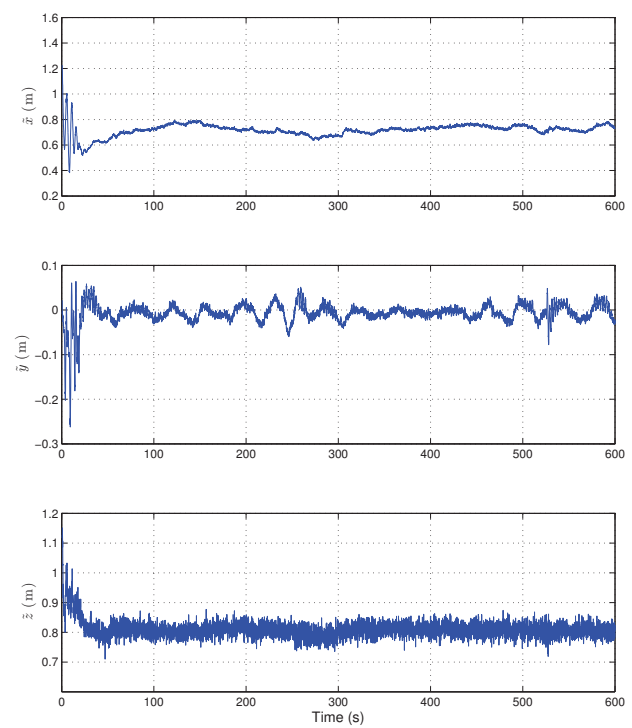

Fig. 9. Relative position of MARES with regard to the target

\section{EXPERIMENTAL RESULTS}

Several tests have been completed in a test tank at INESC TEC/FEUP facilities. The target was placed on the bottom of the tank and MARES was placed so that the target was in its field of view. During experiments, the vehicle was subjected to disturbances induced by water motion in a tank of $4 \mathrm{~m}$ large by $5 \mathrm{~m}$ long and $1.8 \mathrm{~m}$ deep. Moreover, for safety, a cable and a rope were attached to MARES during these operations.

\section{A. Station-keeping at constant depth}

For this experiment, the vertical error was set as a function of the depth thus resulting in control based on the vehicle depth, extracted from a pressure sensor. The intention was to evaluate the capability of MARES to keep the horizontal position with regard to the target. Therefore, the vehicle was expected to dive $30 \mathrm{~cm}$ and continuously track the target in the horizontal plane. The parameter $\delta^{h}$ was set equal to the distance between the vehicle's center of mass and the camera $(0.7 \mathrm{~m})$, in the longitudinal axis. The purpose of this choice is to keep the target centered in the camera's image.

The relative position of the vehicle with regard to the target $\left(\tilde{\eta}_{B}\right)$ is depicted in Fig. 9. As it can be seen, during initial instants there is an oscillation of the relative position estimate. This is caused by the AUV motion, namely by the oscillating rolling and pitching angles as shown in Fig. 10. In fact, longitudinal thrusters induce a rolling moment when they are actuating. This moment grows directly with the force exerted by the thrusters which is particularly large during these instants and decreases after the vehicle reaches the vicinity of the reference. The (small) oscillation in roll is also responsible for the oscillation of $\tilde{y}$ which does not appear to be critical, though.
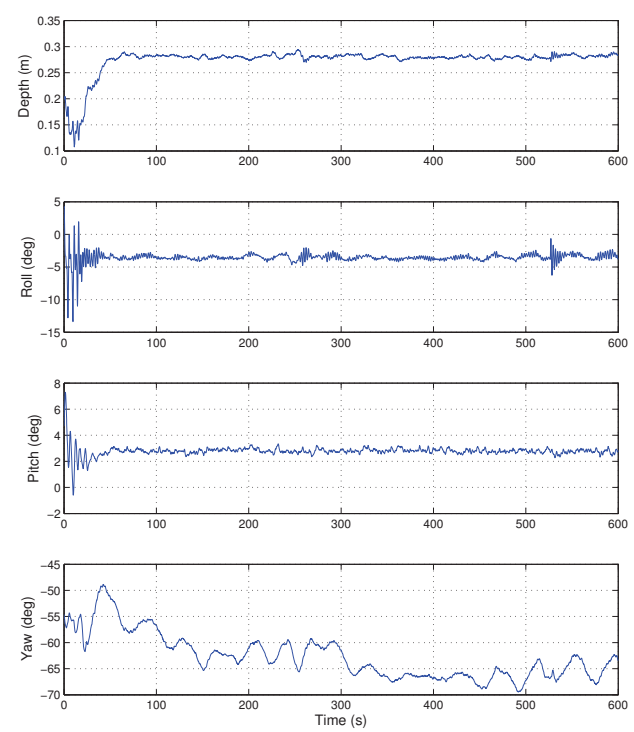

Fig. 10. Depth and angles

It is important to stress that the linear velocity is being estimated based on the thrusters estimated forces only. Hence, it is expectable that the longitudinal axis suffer from steady state errors as seen in $\tilde{x}$ in Fig. 9 (the expected value was $0.7 \mathrm{~m})$. Nevertheless, it is possible to conclude that these errors are below $0.1 \mathrm{~m}$ both in longitudinal and transversal axes.

\section{CONCLusions}

This paper presented a vision-based relative localization method and controllers to position a hovering AUV with regard to a target. Localization includes two main components: features extraction and computation (estimation) of the relative position. Controllers were presented to specifically track a target and results showing the AUV tracking horizontally the target have demonstrated the capabilities of this approach.

\section{APPENDIX}

$$
\begin{aligned}
P_{c}^{h} & =\left[\begin{array}{lll}
1 & 0 & 0
\end{array}\right] \\
P_{u}^{h} & =\left[\begin{array}{lll}
0 & 1 & 0
\end{array}\right] \\
H & =\left[\begin{array}{lll}
1 & 0 & 0 \\
0 & 1 & 0
\end{array}\right] \\
N_{c}^{h} & =\left[\begin{array}{lll}
0 & 0 & 1
\end{array}\right] \\
N_{u}^{h} & =\left[\begin{array}{lll}
1 & 0 & 0 \\
0 & 1 & 0
\end{array}\right] \\
P_{c}^{v} & =\left[\begin{array}{lll}
0 & 0 & 1
\end{array}\right] \\
V & =\left[\begin{array}{lll}
1 & 0 & 0 \\
0 & 1 & 0
\end{array}\right]
\end{aligned}
$$




\section{ACKNOWLEDGEMENT}

A. B. Figueiredo acknowledges the support of the Portuguese Foundation for Science and Technology (FCT) through grant SFRH/BD/81724/2011.

This work has been developed as part of project ENDURE - Enabling Long-Term Deployments of Underwater Robotic Platforms in Remote Oceanic Locations. ENDURE (PT02_Aviso4_0015) benefits from a $€ 218157$ grant from Iceland, Liechtenstein and Norway through the EEA Grants. The aim of the programme PT02 is to achieve a good environmental status, in accordance with the Marine Strategy Framework Directive and one of the expected outputs consists in the strengthening of the capacity of mobile remote monitoring ocean through the introduction of innovative supporting platforms.

This work was partially funded by the project STRONGMAR - STRONGMAR: STRengthening MARritime technology Research Center (H2020-TWINN-2015 (CSA)-692427)
This work is financed by the ERDF European Regional Development Fund through the Operational Programme for Competitiveness and Internationalisation - COMPETE 2020 Programme within project "POCI-01-0145-FEDER-00696", and by National Funds through the FCT Fundao para a Ciłncia e a Tecnologia (Portuguese Foundation for Science and Technology) as part of project UID/EEA/50014/2013.

\section{REFERENCES}

[1] A. Figueiredo, B. Ferreira, and A. Matos, "Tracking of an underwater visual target with an autonomous surface vehicle," in Oceans - St. John's, 2014, Sept 2014, pp. 1-5.

[2] N. Philip and M. Ananthasayanam, "Relative position and attitude estimation and control schemes for the final phase of an autonomous docking mission of spacecraft," Acta Astronautica, vol. 52, no. 7, pp. 511 - 522, 2003. [Online]. Available: http://www.sciencedirect.com/science/article/pii/S009457650200125X

[3] T. I. Fossen, Guidance and control of ocean vehicles. Wiley, 1994.

[4] B. Ferreira, A. Matos, and N. Cruz, "Single beacon navigation: Localization and control of the mares auv," in OCEANS 2010, September 2010, pp. $1-9$. 